\title{
OPEN Circulating tumor DNA (ctDNA) detection is associated with shorter progression-free survival in advanced melanoma patients
}

\author{
Gabriella Taques Marczynski ${ }^{1}$, Ana Carolina Laus ${ }^{1}$, Mariana Bisarro dos Reis ${ }^{1}$, \\ Rui Manuel Reis ${ }^{1,2,3}$ \& Vinicius de Lima Vazquez ${ }^{1,4,5} \bowtie$
}

$B R A F, N R A S$ and TERT mutations occur in more than 2/3 of melanomas. Its detection in patient's blood, as circulating tumor DNA (ctDNA), represents a possibility for identification and monitoring of metastatic disease. We proposed to standardize a liquid biopsy platform to identify hotspot mutations in BRAF, NRAS and TERT in plasma samples from advanced melanoma patients and investigate whether it was associated to clinical outcome. Firstly, we performed digital polymerase chain reaction using tumor cell lines for validation and determination of limit of detection (LOD) of each assay and screened plasma samples from healthy individuals to determine the limit of blank (LOB). Then, we selected 19 stage III and IV patients and determined the somatic mutations status in tumor tissue and track them in patients' plasma. We established a specific and sensitive methodology with a LOD ranging from 0.13 to $0.37 \%$, and LOB ranging from of 0 to 5.201 copies/reaction. Somatic mutations occurred in $17 / 19$ (89\%) patients, of whom seven (41\%) had ctDNA detectable their paired plasma. ctDNA detection was associated with shorter progression free survival $(p=0.01)$. In conclusion, our data support the use of ctDNA as prognosis biomarker, suggesting that patients with detectable levels have an unfavorable outcome.

Melanoma is the most aggressive type of skin cancer due to the high occurrence of metastases and resistance to conventional chemotherapy ${ }^{1}$. Moreover, melanoma rates have been rising for the last decades in predominantly fair-skinned populations and despite the favorable prognosis in early stages, local advanced or metastatic disease are still life-threatening conditions ${ }^{2}$. Currently, with the use of BRAF/MEK inhibitors and immunotherapy, the survival rates for metastatic melanoma have increased substantially. However, only a few patients produce durable responses, while the remainders develop resistance during treatment and experience disease progression at some point ${ }^{3-6}$.

The actual staging system from the American Joint Committee on Cancer (AJCC) includes prognostic and predictive biomarkers for melanoma ${ }^{7}$. However, AJCC only considers anatomical and histological features, which implicates that treatment decision and monitoring decisions are based solely on clinical and imaging findings, since there are no reliable blood-based biomarkers applicable for routine clinical use ${ }^{8,9}$. To rapidly identify metastatic progression and appropriately select patients for more aggressive therapy and avoid unnecessary treatments, there is a need for more tumor biomarkers.

In this context, a candidate biomarker that has been intensively evaluated is the circulating tumor DNA (ctDNA), which makes up part of cell-free DNA (cfDNA) from plasma ${ }^{10,11}$. The cfDNA comprises short fragments of DNA (160-180 base pairs), originated from cell death processes including necrosis and apoptosis and also cellular secretions ${ }^{12}$. The cfDNA concentration varies from 10 to $15 \mathrm{ng}$ per milliliter of blood in healthy individuals, however it increases in certain conditions such as inflammation, exercise, tissue injury and in patients with cancer ${ }^{13}$. The ctDNA is the term for the fraction of cfDNA derived from tumor and therefore carries genetic alterations from the tumor. By the knowledge of tumor specific alterations, it is possible not only to identify

\footnotetext{
${ }^{1}$ Molecular Oncology Research Center, Barretos Cancer Hospital, 1331, Antenor Duarte Villela St, Barretos, SP 14784-400, Brazil. 'Life and Health Sciences Research Institute (ICVS), School of Medicine, University of Minho, Braga, Portugal. ${ }^{3}$ ICVS/3B's - PT Government Associate Laboratory, Braga, Guimarães, Portugal. "Surgery Department of Melanoma, Sarcoma and Mesenchymal Tumors, Barretos Cancer Hospital, Barretos, Brazil. ${ }^{5}$ Barretos School of Health Sciences Dr. Paulo Prata - FACISB, Barretos, Brazil. ${ }^{\square}$ email: viniciusvazquez@gmail.com
} 
molecular changes of the tumor but also to monitoring tumor burden in plasma. Recently, the detection of ctDNA alterations in melanoma patients has gain attention, due to its practical advantages: it is minimally invasive, allows serial collection, and is less prone to limitations such as quality of the material and tumor heterogeneity than traditional tissue biopsies ${ }^{14-16}$. Some relevant findings of this method include the association of ctDNA levels to tumor burden in metastatic melanoma patients and the ability of baseline values to predict outcomes ${ }^{11,17,18}$. The ctDNA variability during melanoma treatment is another possible tool for early assessment of therapy response or resistance $\mathrm{e}^{18-23}$.

The most frequent and explored alterations are BRAF and NRAS mutations, present in $40-60 \%$ and $28 \%$ of patients, respectively ${ }^{24,25}$. In addition, a high frequency of mutations in the promoter region of TERT gene has been found in melanoma patients with a frequency ranging from 50 to $80 \%$ in several studies ${ }^{24,26,27}$, including Brazilian populations ${ }^{28}$. The most frequently described mutations are C228T and C250T, which are mutually exclusive ${ }^{24}$, but can co-occur with BRAF or NRAS mutations and are associated with lower survival ${ }^{28-30}$.

Therefore, we hypothesize that determining these driver mutations in tumor tissue and tracking them in plasma, it could contribute to identify melanoma patients at higher risk for progression. The aims of this study were to standardize a highly sensitive methodology of digital PCR (dPCR) to accurately identify hotspot mutations in BRAF, NRAS, and TERT in plasma and interrogate its feasibility as a liquid biopsy tool for melanoma patients.

\section{Results}

Establishment of optimal conditions of digital PCR. For each BRAF, NRAS, TERT C250T and TERT C228T mutation detection assay, we performed temperature gradients to identify optimal annealing extension temperatures and times. Both $B R A F$ and NRAS assays demonstrated better clustering between populations at $53^{\circ} \mathrm{C}$ of annealing temperature, as shown in Fig. 1a-d. For BRAF mutation assay, the positive control (UACC62$B R A F$ V600E) showed two populations, the double negative droplets and the FAM positive (mutant) droplets, since the cell line harbor the V600E mutation in homozygosis (Fig. 1a). The BRAF negative control (SKMEL103 melanoma cell line), besides empty droplets, presented a population only on the y-axis, being correctly classified as wild type (Fig. 1b). For NRAS assay, the Q61R mutation positive control (SKMEL103) exhibited the mutation in heterozygosis, and therefore showed in the two dimensional plot representation of the four populations: the mutated homozygous droplets in blue, the droplets with the two alleles in orange, the wild type only droplets in green, and the double negative droplets in gray (Fig. 1c). As expected, the wild type control for NRAS (UACC62), presented double negative, and wild type droplets (Fig. 1d).

Due to the design characteristics of the oligonucleotides and amplification target region, both TERT assays required different cycling conditions. For TERT mutation the optimal conditions were under 54 cycles with annealing temperature of $59^{\circ} \mathrm{C}$ (Fig. 1e). The positive control, heterozygous for TERT C250T mutation (SIHA cervical cell line), presented the four populations while negative control (HS587T breast cell line) presented double negative and the wild type droplets population (Fig. 1e, f, respectively). For the TERT C228T assay, despite extensive optimization attempts, the assay did not perform well in QX200 Droplet Digital PCR (Bio-Rad Laboratories, USA), presenting probe cross-reactivity. Instead, we benefit from testing TERT C228T in Quant Studio 3D (ThermoFisher Scientific, USA) equipment, using 54 cycles with annealing temperature of $55^{\circ} \mathrm{C}$ (according to the manufacturer's recommendation). The positive control for TERT C228T (HS587T) had a similar distribution to the other heterozygous cell line previously demonstrated, with four populations (Fig. 1g). The wild type control (SIHA) presented some false-positive reactions (Fig. 1h).

Determination of limit of blank (LOB), limit of detection (LOD) and linearity evaluation. The limits of blank (LOBs) for each assay were calculated as described and results are summarized in Supplementary Table S1. For BRAF assay, since no mutant copies were observed in any control, the LOB was 0 copies/reaction. For NRAS assay, we found a LOB of 2.13 copies/reaction. Both TERT assays had a higher level of false-positive rates with LOBs of 3.995 and 5.201 copies/reaction for C228T and C250T, respectively (Supplementary Table S1).

The next step was to evaluate the linearity of assays and determine the analytical sensitivity of each assay. Using replicates of six dilutions of mutant in wild type DNA background, (Fig. 2). A high linearity between the expected and the measured fraction of mutant DNA was observed with a $\mathrm{R}^{2}=0.9986$ for BRAF (Fig. 2a), $\mathrm{R}^{2}=0.9982$ for NRAS (Fig. 2b), $\mathrm{R}^{2}=0.996$ for TERT C228T (Fig. 2c) and $\mathrm{R}^{2}=0.9989$ for TERT C250T (Fig. 2d).

The lowest concentration sample dilution was used to determine the LOD of assays, calculated as described by Armbruster and Pry ${ }^{31}$, varied from 3.71 to 9.76 copies/reaction with fractional abundance of $0.13 \%$ to $0.37 \%$ (Supplementary Table S1).

Patient demographics features and detection of baseline ctDNA. BRAF, NRAS, and TERT somatic mutations were evaluated in patient's tumor tissue as part of clinical routine (Fig. 3). From 19 patients analyzed, 17 (89\%) had at least one somatic mutation. Twelve patients had BRAF mutations $(63.2 \%-10$ patients: V600E, 2 patients-V600K), while two patients (10.5\%) presented NRAS mutation (1 patient-Q61H; 1 patient-Q61R) and 14 patients had TERT alterations (74\%) (7 patients-C228T; 7 patients-C250T). Most patients $(\mathrm{n}=11)$ presented more than one gene altered, and as expected $B R A F$ and NRAS were mutually exclusive. Two patients were triple negative for selected genes (IDs 4 and 14) (Fig. 4).

Clinical information of the nineteen patients are summarized in Fig. 4. Overall, 12 (63.2\%) patients were female and 7 (36.8\%) were male, the ages ranged from 34 to 79 years, with a mean of 61 years, $15(79 \%)$ had distant metastases (stage IV) and 4 (21\%) presented only lymph node involvement (stage III). The most frequent histological subtypes were acral and nodular melanoma (5 patients each). Two patients (IDs 4 and 9) had only brain metastasis with unknown primary site and 3 patients had no information on the primary lesion, being 
a)

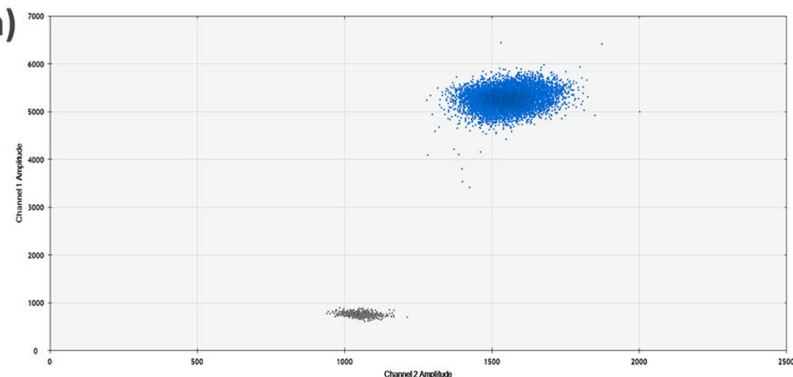

c)

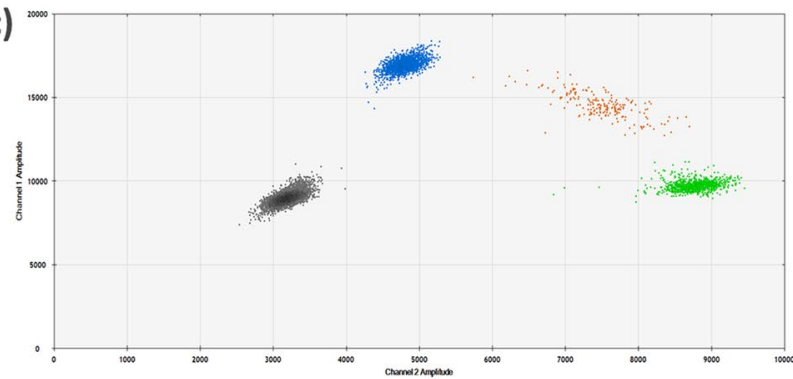

e)

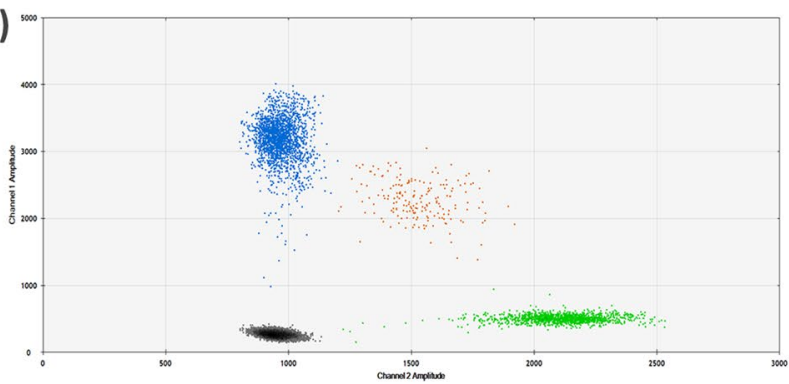

g)

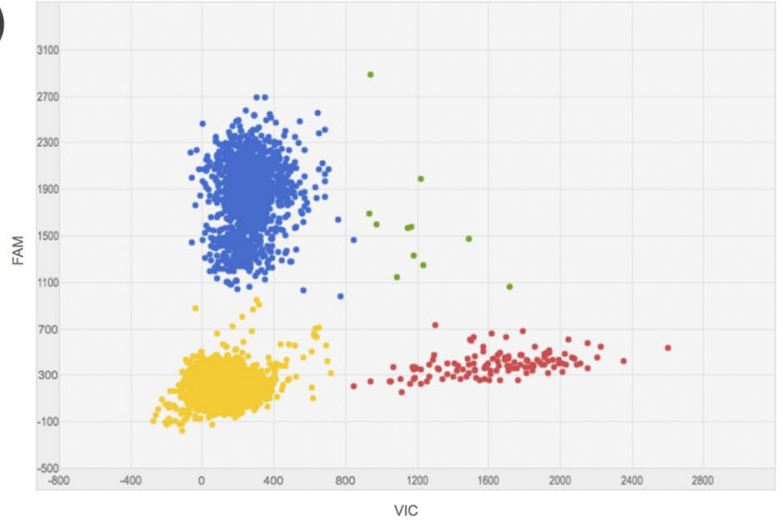

b)

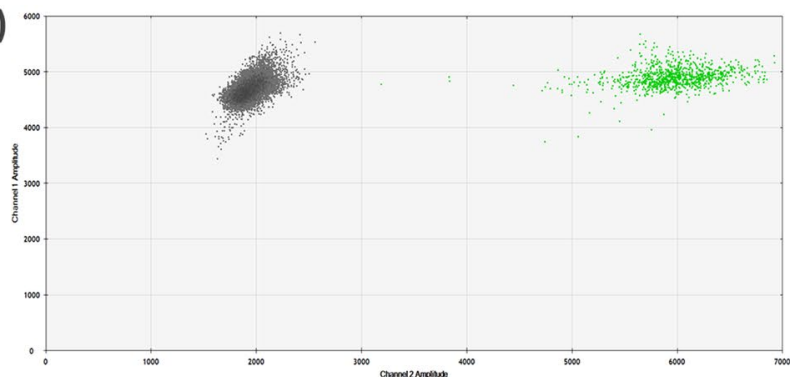

d)

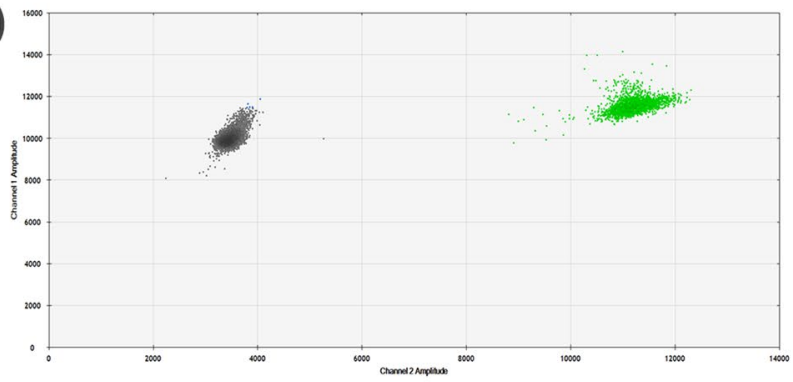

f)

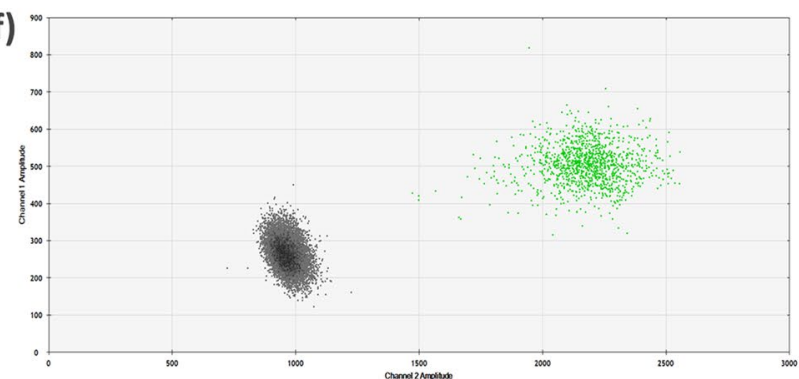

EAM: 0 VIC: 3591 FAM+VIC: 4 UNDETERMINED:0 NO-AMP: 12702

h)

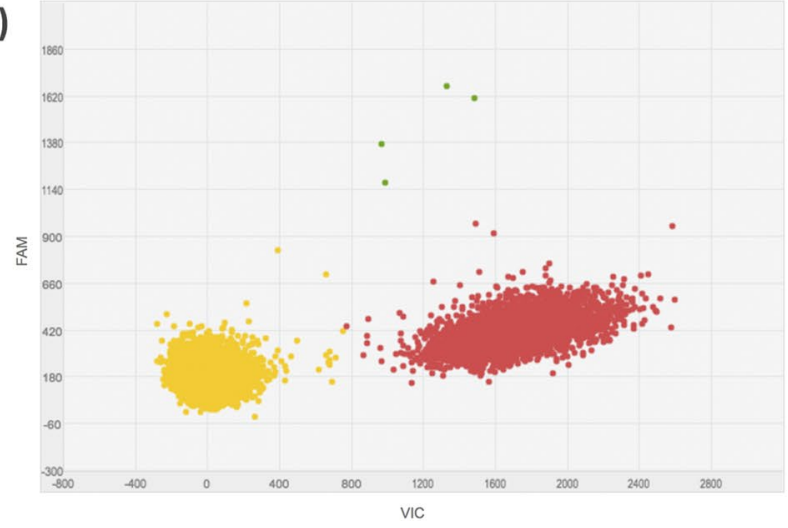

Figure 1. Scatter plots of $B R A F(\mathbf{a}, \mathbf{b}), N R A S(\mathbf{c}, \mathbf{d})$ and $T E R T(\mathbf{e}, \mathbf{h})$ assays. In all plots, the $\mathbf{y}$-axis represents mutant channel and the $\mathrm{x}$-axis represents the wild type channel. (a) $B R A F+\mathrm{UACC} 62$ cell line. (b) $B R A F-$ SKMEL103 cell line. (c) NRAS + SKMEL 103 cell line. (d) NRAS- UACC62 cell line. (e) TERT C250T + SIHA cell line. (f) TERT C250T- HS587T cell line. (g) TERT C228T + HS587T cell line on QuantStudio3D platform. (h) TERT C228T- SIHA cell line, presenting a small positive double population in the upper right corner (green), which in this case was considered false positive.

classified as an unknown histological subtype. Regarding the Breslow thickness of the primary lesion, both the mean and the median thickness of the group were around $7.5 \mathrm{~mm}$, so that the patients were grouped according to this value, above and below (Fig. 4). Finally, 5 patients died and 14 are still being followed up.

ctDNA could be detected in seven (41\%) of the 17 patients that had at least one mutation identified in the tumor tissue. Quantification of mutant targets (copies/reaction) for each assay is described in Supplementary Table 1 (Table S2). Analyzing by gene, BRAF mutations were detected in plasma from in 5 of 12 cases (41.6\%), in 1 of 2 cases of NRAS positive (50\%), and in 5 of 14 TERT positive mutations (35.7\%). From 12 patients that carried two genes mutated on tumor tissue, both alterations were detected in plasma in 4 cases (33.3\%), in 7 
a

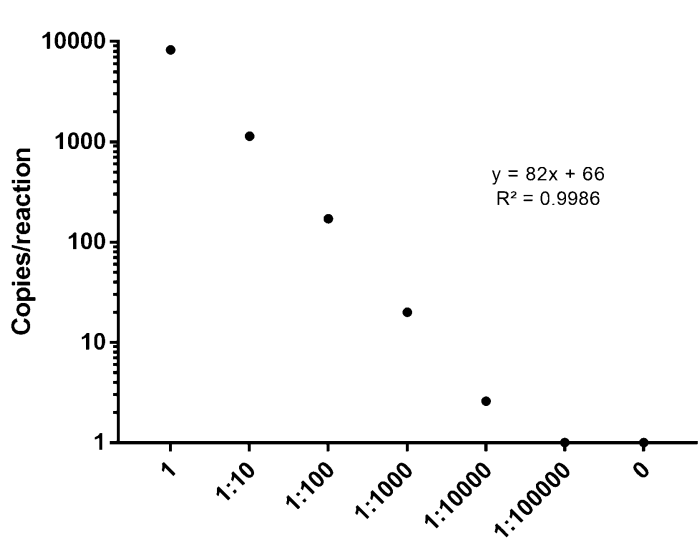

Mutant:WT ratio

C

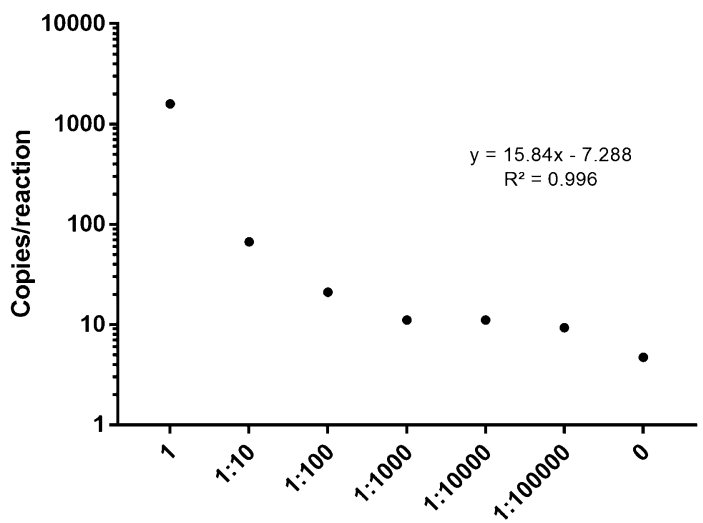

Mutant:WT ratio b

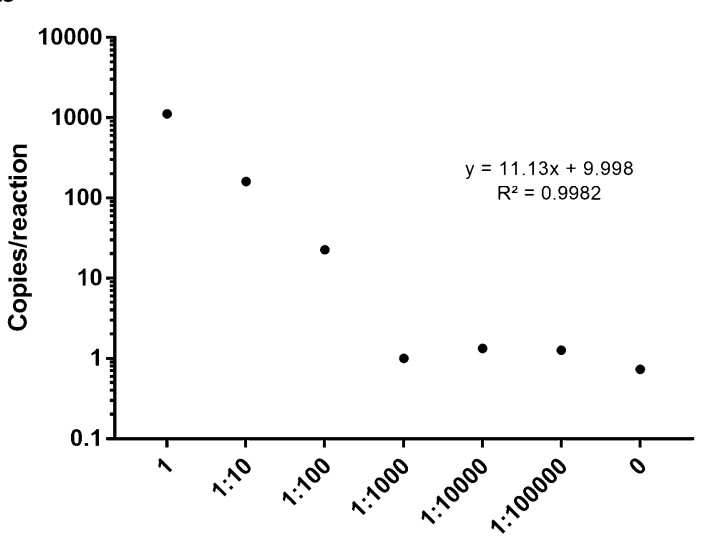

Mutant:WT ratio

d

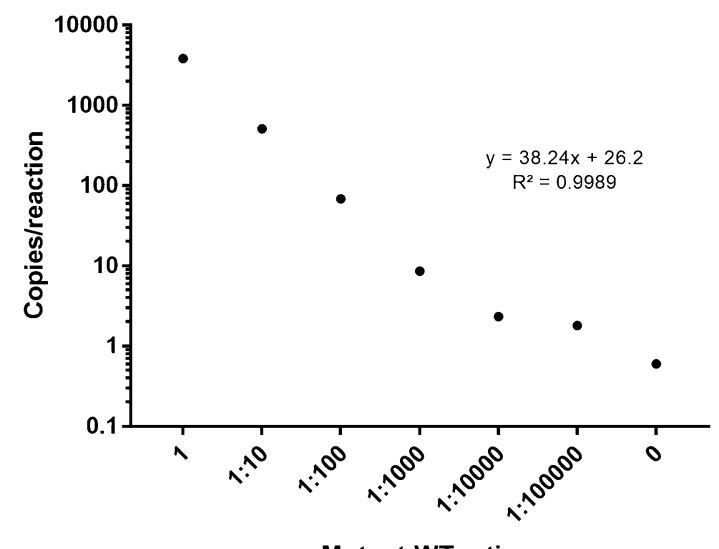

Figure 2. Serial dilution of mutant DNA from cell lines in a wild type background DNA. The number mutant DNA copies determined by dPCR were plotted against the corresponding dilutions. (a) Dilution of DNA from cell line UACC62 harboring BRAF V600E mutation. (b) Dilution of DNA from cell line SKMEL 103 harboring NRAS Q61R mutation. (c) Dilution of DNA from cell line HS587T harboring TERT C228T mutation. (d) Dilution of DNA from cell line SIHA harboring TERT C250T mutation.

\section{BRAF $\quad 63 \%$}

NRAS $\quad 11 \%$

TERT

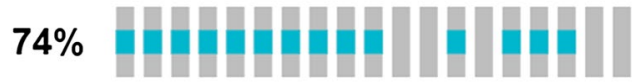

Missense Mutation (putative driver) Promoter Mutation No alterations

Figure 3. Somatic mutation profile determined on FFPE samples. Patients are classified according to presence of mutations in BRAF, NRAS, TERT or TRIPLE negative/wild type.

patients, neither mutation was detected in ctDNA, and 1 patient had only one of the mutations observed (8.3\%). Patients without mutation in the FFPE tissue did not exhibited mutation in the plasma (Fig. 4).

Association of ctDNA and patients's prognostic factors. We grouped the 17 patients according to ctDNA detection in plasma: detectable $(n=7)$ and undetectable $(n=10)$. The association between the clinical features of the 17 patients and ctDNA detection is shown in Table 1. Of the patients with detectable ctDNA, 5 $(71 \%)$ were younger patients (<61 years). It was observed a tendency between Breslow thicknesses of primary 


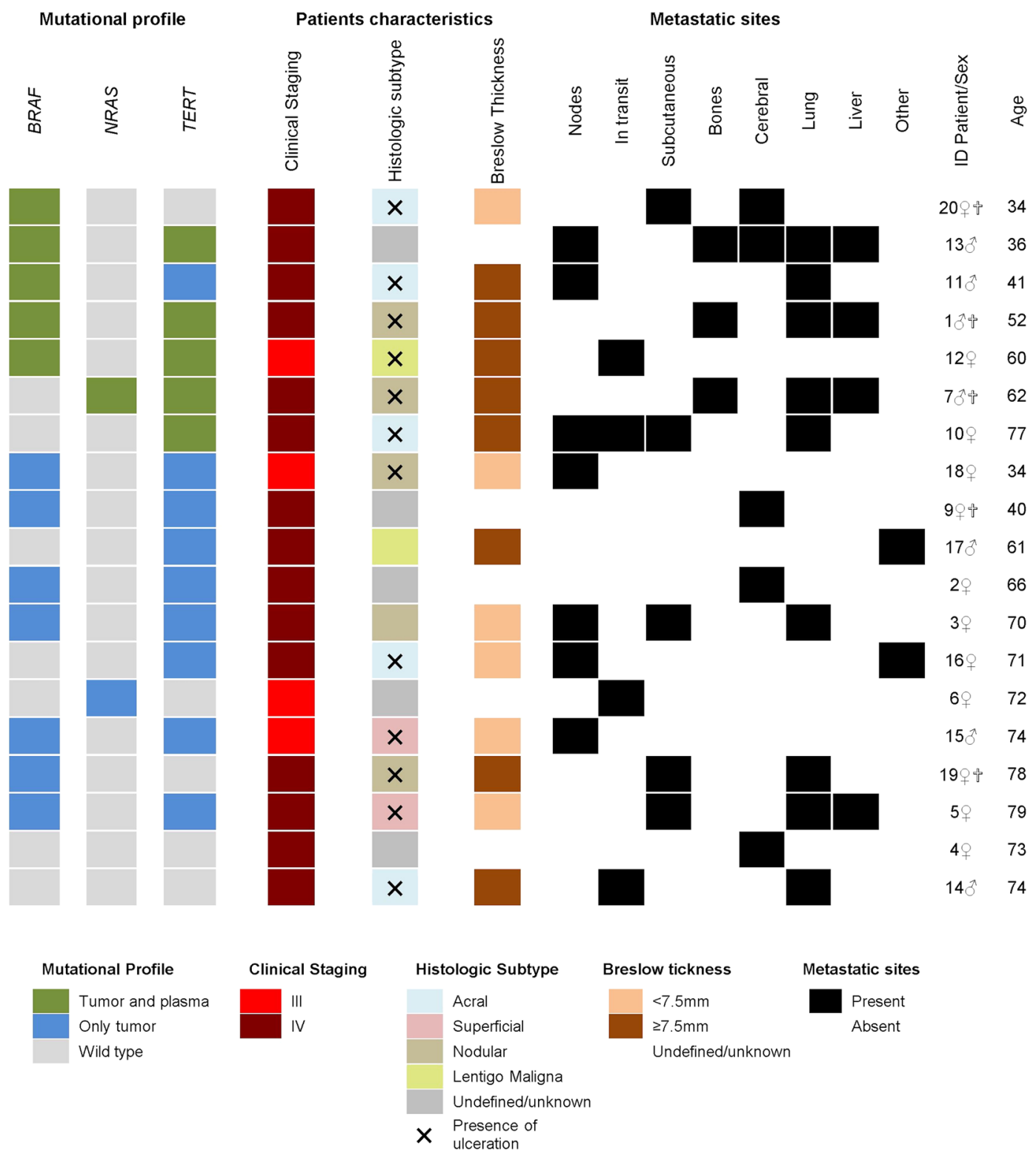

Figure 4. Overview of patient's mutational profile, characteristics, and site of metastasis. Patients are ordered according to ctDNA detection.

lesions greater than $7.5 \mathrm{~mm}$ with detectable ctDNA, yet it was not significant. Also, a higher frequency of detectable ctDNA was observed in patients with more than one metastasis site. Individuals with isolated central nervous system (CNS) metastases had no detectable ctDNA (IDs 2 and 9).

Association of ctDNA and patient outcome. The median follow-up of patients was 130 days, ranging from 7 to 253. Patients who had detectable levels of ctDNA had a shorter progression-free survival when compared to patients with undetectable mutation $(p=0.01)$ (Fig. 5). The median time in days for progression in patients with circulating tumor DNA was 50 days, and in patients without circulating tumor DNA it was 146 days. Patients with detectable ctDNA had significantly increased risk of progression compared with those with undetectable ctDNA (HR: 6.38; 95\% CI 1.26-32.1; $p=0.025$ ).

\section{Discussion}

The emergence of target therapy and immunotherapy has contributed significantly to the survival of patients with metastatic melanoma ${ }^{4}$. However, melanoma lacks prognostic and predictive biomarkers, which implicates on the challenges in select patients with a poorer prognosis that will benefit from a more aggressive therapy and for early identification of disease progression.

Here, we validated a dPCR approach that detects the most frequent melanoma alterations in cfDNA in a real-world context. We demonstrated that these specific mutations are present in tumor tissue of $89 \%$ of our 


\begin{tabular}{|c|c|c|c|}
\hline Variables & Detectable & Undetectable & $p$ value \\
\hline \multicolumn{4}{|l|}{ Sex ${ }^{a}$} \\
\hline Female & $3(27 \%)$ & $8(73 \%)$ & \multirow{2}{*}{0.162} \\
\hline Male & $4(67 \%)$ & $2(33 \%)$ & \\
\hline \multicolumn{4}{|l|}{ Age $^{\mathrm{a}, \mathrm{b}}$} \\
\hline$<61$ years & $5(71 \%)$ & $2(29 \%)$ & \multirow{2}{*}{0.058} \\
\hline$\geq 61$ years & $2(20 \%)$ & $8(80 \%)$ & \\
\hline \multicolumn{4}{|l|}{ Clinical stage $^{\mathrm{a}}$} \\
\hline III & $1(25 \%)$ & $3(75 \%)$ & \multirow{2}{*}{0.603} \\
\hline IV & $6(46 \%)$ & $7(54 \%)$ & \\
\hline \multicolumn{4}{|l|}{ Histological subtype $^{a}$} \\
\hline Acral & $3(75 \%)$ & $1(25 \%)$ & \multirow{5}{*}{0.435} \\
\hline Superficial & $0(0 \%)$ & $2(100 \%)$ & \\
\hline Nodular & $2(40 \%)$ & $3(60 \%)$ & \\
\hline Lentigo Maligna & $1(50 \%)$ & $1(50 \%)$ & \\
\hline Undefined/unknown & $1(25 \%)$ & $3(75 \%)$ & \\
\hline \multicolumn{4}{|l|}{ Breslow thickness ${ }^{\text {a.c }}$} \\
\hline$<7.5 \mathrm{~mm}$ & $1(17 \%)$ & $5(83 \%)$ & \multirow{3}{*}{0.103} \\
\hline$\geq 7.5 \mathrm{~mm}$ & $5(71 \%)$ & $2(29 \%)$ & \\
\hline Undefined/unknown & $1(25 \%)$ & $3(75 \%)$ & \\
\hline \multicolumn{4}{|l|}{ Ulceration $^{\mathrm{a}}$} \\
\hline Absent & $0(0 \%)$ & $2(100 \%)$ & \multirow{3}{*}{0.267} \\
\hline Present & $6(55 \%)$ & $5(45 \%)$ & \\
\hline Undefined/unknown & $1(25 \%)$ & $3(75 \%)$ & \\
\hline \multicolumn{4}{|c|}{ Site of distant metastasis ${ }^{a}$} \\
\hline M0 & $1(25 \%)$ & $3(75 \%)$ & \multirow{5}{*}{0.723} \\
\hline M1a & $0(0 \%)$ & $1(100 \%)$ & \\
\hline M1b & $1(33 \%)$ & $2(67 \%)$ & \\
\hline M1c & $3(60 \%)$ & $2(40 \%)$ & \\
\hline M1d & $2(50 \%)$ & $2(50 \%)$ & \\
\hline \multicolumn{4}{|c|}{ Number of distant metastasis ${ }^{\mathrm{add}}$} \\
\hline 1 Sites of metastasis & $1(20 \%)$ & $4(80 \%)$ & \multirow{2}{*}{0.266} \\
\hline$>2$ Sites of metastasis & $5(63 \%)$ & $3(37 \%)$ & \\
\hline \multicolumn{4}{|c|}{ Number of somatic mutations ${ }^{a}$} \\
\hline One & $2(29 \%)$ & $5(71 \%)$ & \multirow{2}{*}{$>0.999$} \\
\hline Two & $5(50 \%)$ & $5(50 \%)$ & \\
\hline \multicolumn{4}{|l|}{ Mutational status $^{\mathrm{a}}$} \\
\hline$B R A F$ & $1(50 \%)$ & $1(50 \%)$ & \multirow{5}{*}{0.685} \\
\hline NRAS & $0(0 \%)$ & $1(100 \%)$ & \\
\hline TERT & $1(33 \%)$ & $2(67 \%)$ & \\
\hline$B R A F+T E R T$ & $4(40 \%)$ & $6(60 \%)$ & \\
\hline NRAS + TERT & $1(100 \%)$ & $0(0 \%)$ & \\
\hline
\end{tabular}

Table 1. Association of ctDNA with melanoma patient's clinical features $(n=17) .{ }^{a}$ Analysis by Fisher's exact test expressed as absolute (n) and relative (\%) frequencies; ${ }^{b}$ Categories defined from mean; ${ }^{c}$ Categories defined from median; ${ }^{\mathrm{d}}$ Only stage IV patients.

patients, and taking together, mutations could be detected in plasma from seven of seventeen patients (41\%). Briefly, ctDNA was detected in 5 of 12 cases $B R A F$ positive (41.6\%), in 1 of 2 cases NRAS positive (50\%), and in 5 of 14 TERT positive mutations (35.7\%).

Liquid biopsy appears as a minimally invasive model, low-cost, sensitive, reproducible and with a wide variety of applications in personalized medicine for melanoma through analysis of circulating tumor DNA ${ }^{11,25,32}$. In attempt to implement liquid biopsy in a clinical setting, our group sought to standardize a platform to evaluate hotspot mutations in melanoma patients. In addition to BRAF and NRAS alterations, which are the most explored genes in most studies ${ }^{33}$, we included the TERT gene, since mutations in its promoter region have been described by its high frequency in melanoma patients ${ }^{27-29}$. The dPCR methodology was chosen due to its high sensitivity and accuracy, especially in the detection of rare alleles at low frequencies, that best fits in this context ${ }^{34,35}$. The major disadvantage of dPCR is that, generally, only one target per test can be evaluated in dPCR, which implicate higher cost and time consuming, when analyzing a large number of genes. Even that multiplex assays have 


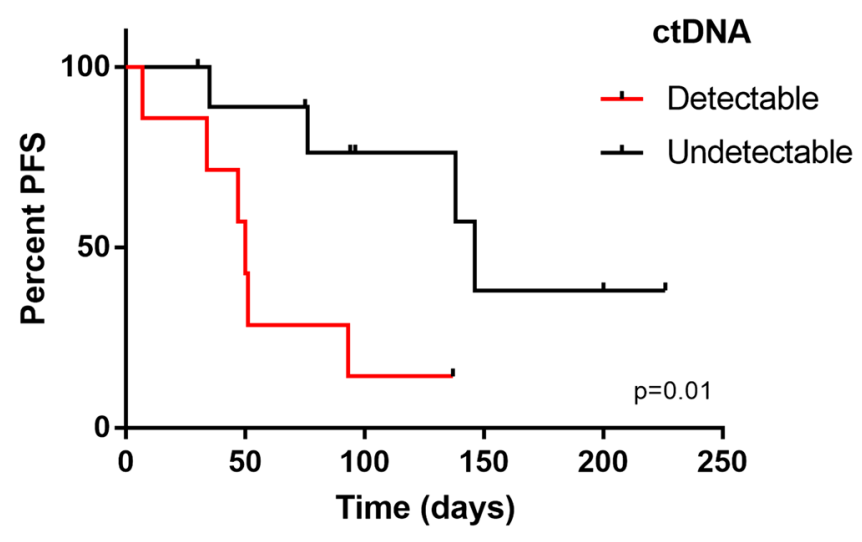

Figure 5. Kaplan-Meier curve Association between detectable ctDNA and progression free survival (median PFS, 50 days for detectable ctDNA and 146 days for undetectable ctDNA, $p=0.014$ ).

been developed recently, there is a limitation in number of targets to be included, due to the necessity that PCR conditions must be the same for all targets. Besides that, the majority of dPCR systems have only two channels of fluorescence detection (FAM and VIC/HEX), which can limit the design of assays with many targets.

Other methods of ctDNA detection can be used, as Next-generation sequencing (NGS). NGS can provide not only the ability to detect genome-wide DNA variation but also the use of multiple targeted panels ${ }^{36}$. The use of these panels has lower cost compared to whole-genome-sequencing (WGS) and whole-exome sequencing (WES) and also offers the advantage of reducing the DNA input and still achieving a high sensitivity. However, the detection of rare alleles at a low frequency requires a very high coverage $(>10.000 \times)$, which increases sequencing $\operatorname{cost}^{37}$. In addition, there is an important limitation in the analysis of TERT promoter mutations in NGS panels. Despite validated for FFPE NGS panels ${ }^{38}$, TERT promoter mutations have not performed well for cfDNA in NGS custom panels ${ }^{39,40}$ due to its high GC content, and so, it is mostly described in droplet digital PCR approaches ${ }^{41,42}$. Furthermore, BRAF and NRAS mutations are mutually exclusive, as well as TERT C250T and C228T, and then, a maximum of two mutations will be tested in plasma for each melanoma patients, making feasible the dPCR approach proposed in this study.

We detected plasma ctDNA in seven of seventeen patients who had at least one somatic mutation in tumor tissue. This is in agreement with three other similar studies using ddPCR, which indicated that ctDNA detection rates are associated with tumor burden ${ }^{18,23,43}$. McEvoy et al ${ }^{18}$ demonstrated a detection rate of $71.8 \%$ in a cohort of 32 stage IV patients. In keeping with the relationship to burden, detection rates fell to $12 \%$ in lower stage patients (EC II and III) ${ }^{23}$. The same group showed a detection rate of $53 \%$ at baseline in stage IV patients that undergone treatment with PD1 inhibitors ${ }^{22}$. Data from Braune and colleagues, showed an even higher detection rate of $87.1 \%$ in a group of 62 patients that comprised 50 stage IV and 12 stage III melanoma patients ${ }^{43}$. The fact that our group comprised both stage III and IV indicates that our detection rate was similar to other studies examining these stages. However, the study from McEvoy, demonstrated that ctDNA detection not only depends on the clinical stage, but also on the metabolic tumor burden (MTB) and metabolic tumor volume (MTV) accessed by 18F-labeled fluorodeoxyglucose positron emission tomography/computed tomography, as ctDNA was undetectable in all patients with an MTB value of $\leq 10^{18}$.

Another important aspect associated to ctDNA detection is the volume of plasma processed and the input of DNA in dPCR reaction, as the screening of small amounts of sample may not always represent the true mutant content, mainly when they are present in a few copies in our sample. The high detection rate from Braune et al. might be associated to the higher input screened. Despite using $2 \mathrm{ml}$ of plasma they tested samples in 4 replicates ${ }^{43}$. Corless and colleagues screened 12 replicates from plasma samples for TERT evaluation in ddPCR ${ }^{42}$. Some other studies isolated DNA from a larger volume of plasma, up to $5 \mathrm{~mL}^{41,44}$. We presume the most important limitation of our assay was the fact that it was possible to used only $2 \mathrm{~mL}$ of plasma and $10 \mathrm{ng}$ per reaction, which generated a higher LOB and, consequently LOD. Higher cut-offs implicates in lower sensitivity. It can have affected the accuracy of our experiment in diagnostic sensitivity, but it demonstrated to be important for determining patient prognosis.

TERT mutation occurs in up to $80 \%$ of cutaneous melanomas, which makes this gene extremely important to be part of the panel proposed ${ }^{26-29}$. However, it is established that the high GC content (approximately $80 \%$ ) in the promoter region of the gene imposes difficulties in the design of an efficient and specific PCR assay to detect and quantify mutations in this region ${ }^{42}$. This was a technical challenge with TERT C228T mutation. The assay chosen in this study was designed by ThermoFisher Scientific company, but even after several approaches, it was not possible to standardize the assay in the Bio-Rad platform. However, the analysis of this mutation in QuantStudio 3D systems achieved reliable results, as demonstrated by our results both with cell lines and plasma samples.

Despite preliminary, our data suggest an association between ctDNA and worse prognosis (HR: $6.38 ; 95 \%$ CI $1.26-32.1 ; p=0.025)$, as previously described ${ }^{11,18,19,21-23,41,45-47}$. From patients with detectable ctDNA, $85 \%$ of patients were stage IV, whilst the only stage III patient (ID 12) progressed in 50 days. It is in accordance to other studies that show that the disease burden influences the detection of $\mathrm{ctDNA}^{17,18}$. Also, as reported in literature, 
ctDNA was not detected in patients with exclusive central nervous system metastases, a fact that could be explained by the presence of the blood-brain barrier, which prevents the detection of circulating DNA ${ }^{21}$.

\section{Conclusion}

Our study demonstrates the impact of ctDNA detection in patient prognosis, in which patients with detectable levels have a worse outcome. Further studies should be done to extend and validate these findings. Moreover, a longitudinal study with several samplings will also allow evaluating the variations in ctDNA levels in patients during the treatment to determine the clinical benefit of the therapies.

\section{Materials and methods}

Patient recruitment and study design. We selected naïve-treated melanoma patients with advanced clinical stage III and IV, admitted at Melanoma and Sarcoma Department of Barretos Cancer Hospital from March to November of 2018, or had a follow up appointment during this period. Formalin-fixed paraffinembedded (FFPE) and peripheral blood samples from patients were collected prospectively at baseline. The present study was approved by the Human Research Ethics Committee of the Barretos Cancer Hospital (1465/2017) and all participants gave their informed written consent. All methods were carried out in accordance with relevant guidelines and regulations.

Identification of somatic mutation. Somatic mutation of hotspot regions of BRAF, NRAS, and TERT genes was determined on formalin-fixed paraffin-embedded (FFPE) tissue available from Pathology Department. Detection of mutation in BRAF and NRAS were performed using the TruSight Tumor 15 panel (Illumina, USA) of next generation sequencing (NGS) as standardized in our institution ${ }^{48}$. For TERT, mutation analysis was performed by Sanger Sequencing ${ }^{28}$, or dPCR methodology in those cases that DNA quality did not allow a reliable result from sequencing.

Plasma processing and cfDNA isolation. Between 5 and $10 \mathrm{~mL}$ of whole blood were collected in a tube containing EDTA and processed within four hours after collection for plasma separation. To separate plasma from buffy coat, centrifugation was done at $3500 \mathrm{rpm}$ for $10 \mathrm{~min}$ and then plasma was stored in $1 \mathrm{~mL}$ aliquots in $-80^{\circ} \mathrm{C}$ freezer until DNA isolation. cfDNA was isolated from $2 \mathrm{~mL}$ of the plasma with the commercial QIAamp Circulating Nucleic Acid Kit (Qiagen, Germany), according to the manufacturer's protocol and eluted in $35 \mu \mathrm{L}$ of ultrapure water, quantified using the dsDNA High Sensitivity Assay Kit and Qubit 2.0 Fluorometer (ThermoFisher Scientific, USA) and stored at $-20^{\circ} \mathrm{C}$.

Assays design and Digital PCR platforms. All assays are based on the principle of genotyping, with primer pair delimiting the region of interest and there are two fluorescence/color-labeled probes, one detecting the mutated allele (FAM) and the other one, the wild type allele (HEX/VIC). For BRAF and NRAS, commercial Bio-Rad assays were used (ddPCR BRAF V600 Screening Kit 12001037, capable of detecting mutations in V600E/K/R and ddPCR NRAS Q61 Screening Kit 12001006, capable of detecting mutations in Q61K/L/R/H $183 \mathrm{~A}>\mathrm{T} / 183 \mathrm{~A}>\mathrm{C}$ ). For mutation evaluations in TERT promoter region, TaqMan (ThermoFisher Scientific, USA) assays were used for each mutation, including Hs000000092 (ThermoFisher Scientific, USA) commercial assay for TERT C228T mutation and Hs000000093 (ThermoFisher Scientific, USA) commercial assay for C250T mutation.

BRAF, NRAS and TERT C250T mutations were evaluated in QX200 Droplet Digital PCR (ddPCR) system (Bio-Rad Laboratories, USA). For ddPCR reaction mixture, $1 \times$ ddPCR Supermix for Probes (No dUTP), $1 \times$ of specific assay and a volume from 1 to $9 \mu \mathrm{L}$ of DNA were mixed, depending on the concentration of the sample. The final volume of $20 \mu \mathrm{L}$ of the reaction, together with $70 \mu \mathrm{L}$ of the Droplet Generation Oil for Probes, was submitted to the QX200 Droplet Generator. PCR reactions were performed in C1000 Touch Thermal Cycler with 96-Deep Well Reaction, droplets were read in the QX200 Droplet Reader and analyzed using Quanta Soft software (Bio-Rad Laboratories, USA). The TERT C228T assay was performed on the Quant Studio 3D platform (ThermoFisher Scientific, USA), after a series of optimization that failure in Bio-Rad platform. For the reaction, $1 \times$ Quant Studio 3D Digital PCR Master Mix v2, $1 \times$ of the specific assay and 1-6.6 $\mu \mathrm{L}$ of DNA were mixed according to the concentration of the sample. The final volume of $14.5 \mu \mathrm{L}$ was applied directly to the Quant Studio 3D Digital PCR 20 K Chip v2, and PCR reaction occurred in the Pro Flex thermal cycler using the chip-specific adapter. The chip was inserted into Quant Studio 3D for fluorescence reading and then analyzed by Analysis Suite software (ThermoFisher Scientific, USA).

Validation, optimization, and determination of linearity of DNA amplification. To determine assay's ability to differentiate mutation positive from mutation negative samples, we tested commercial tumor cell lines whose mutational status are known (Table 2). We evaluated optimal amplification conditions for each assay by testing $20 \mathrm{ng}$ of DNA to a range of temperatures above and below the melting temperature in order to choose the one who provides the optimal cluster separation. Next, a serial dilution containing a mutant ratio of $1: 10,1: 100,1: 1000,1: 10,000$ and 1:100,000 in a background of wild type DNA ( $\mathrm{n}=$ three independent replicates) were performed to evaluate the performance and analytical sensitivity of the assays. For this experiment, we tested $10 \mathrm{ng}$, which is the expected yield for testing samples (cfDNA). Sensitivity was defined as the lowest concentration likely to be reliably distinguished from blank samples.

For the evaluation of assay linearity we compared the concentration in copies/reaction to the expected serial dilution with a linear regression. 


\begin{tabular}{|c|c|c|}
\hline & Source & Mutation \\
\hline $\mathrm{UACC}^{\mathrm{a}}{ }^{\mathrm{a}}$ & Melanoma & $B R A F$ V600E $(\mathrm{c} .1799 \mathrm{~T}>\mathrm{A})$ \\
\hline SKMEL $103^{\mathrm{a}}$ & Melanoma & NRAS Q61R (p.Gln61Arg) \\
\hline SIHA $^{\mathrm{b}}$ & Cervical squamous cell carcinoma & $T E R T-146 \mathrm{G}>\mathrm{T}(\mathrm{C} 250 \mathrm{~T})$ \\
\hline HS587 $^{\mathrm{b}}$ & Breast carcinoma & $T E R T-124 \mathrm{C}>\mathrm{T}(\mathrm{C} 228 \mathrm{~T})$ \\
\hline
\end{tabular}

Table 2. Description of tumor cell lines used for validation of commercial assays. ${ }^{\mathrm{a}}$ Cell lines kindly provided by Dr. Silvya Stuchi Maria Engler-University of Sao Paulo. ${ }^{\mathrm{b}}$ Cell lines acquired from ATCC.

Determination of limit of blank (LOB) and limit of detection (LOD). In order to determine the false positive rate for each assay and accurately determine the limit of detection, we analyzed cell-free DNA extracted from plasma donated by 20 healthy volunteers (blank samples). The LOB was calculated as described by Armbruster and Pry ${ }^{31}$, where: $\mathrm{LOB}=$ meanblank $+1.645^{\star}$ SDblank.

After establishing the LOB, we calculated the lowest analyte concentration likely to be reliably distinguished from the background (LOD) as follow: $\mathrm{LOD}=\mathrm{LOB}+1.645^{*}(\mathrm{SD} \text { low concentration sample })^{31}$.

Data analysis of patient samples and positive calls. PCR reactions for patient samples were performed using a maximum input of $10 \mathrm{ng}$ of cfDNA. Each run included mutant positive controls, wild type controls, and non-template controls.

For quality analysis, the overall accepted events/reading should be above 10,000 according to manufacturer's protocol. To determine positive droplets, the threshold was set examining the three controls wells using the 2D plot. Samples were classified as mutated when number of copies/reaction exceeded the LOB calculated for that assay.

The number of mutated DNA copies per reaction was used to calculate copies per $\mathrm{mL}$ of plasma using the following equation:

Copies $/ \mathrm{mL}$ of plasma $=\mathrm{C}^{\star} \mathrm{EV} / \mathrm{TV} / \mathrm{PV} . \mathrm{PV}=$ Volume of plasma used for $\mathrm{cfDNA}$ extraction $(\mathrm{mL})$ $\mathrm{EV}=$ Volume in which cfDNA was eluted $(\mu \mathrm{L}), \mathrm{TV}=$ Volume of cfDNA added to the PCR reaction $(\mu \mathrm{L}), \mathrm{C}=\mathrm{cop}-$ ies/reaction (data derived from QuantaSoft or Analysis Suite softwares).

Statistical analysis. Association between patient detection of ctDNA (undetectable/detectable) and patient's characteristics (gender, age, AJCC stage, histological classification, primary melanoma Breslow and ulceration, site of metastasis, as well as mutational status) were performed using a Fisher's exact test. Progression free survival (PFS) was calculated as the time from the date of collection/inclusion to the date of the first disease progression (PD) clinically defined or censored with stable disease at the most recent visit. Median PFS was calculated using the Kaplan-Meier method and compared using the log-rank test. A Cox proportional hazards regression analysis was performed to examine association of ctDNA detection with PFS. Statistical analysis was performed using the IBM SPSS Statistics for Windows version 20.0.

All results were considered significant when $p$ value was $<0.05$. Clinical, demographic, histopathological, and treatment-related data, as well as disease progression or relapse data were prospectively collected using a questionnaire and medical records and stored in an electronic database (REDCap).

Received: 28 May 2020; Accepted: 20 October 2020

Published online: 29 October 2020

\section{References}

1. Eggermont, A. M. \& Kirkwood, J. M. Re-evaluating the role of dacarbazine in metastatic melanoma: what have we learned in 30 years?. Eur. J. Cancer 40, 1825-1836. https://doi.org/10.1016/j.ejca.2004.04.030 (2004).

2. Bray, F. G. et al. Global cancer statistics 2018: GLOBOCAN estimates of incidence and mortality worldwide for 36 cancers in 185 countries. CA Cancer J. Clin. https://doi.org/10.3322/caac.21492 (2018).

3. Luke, J. J., Flaherty, K. T., Ribas, A. \& Long, G. V. Targeted agents and immunotherapies: optimizing outcomes in melanoma. Nat. Rev. Clin. Oncol. 14, 463-482. https://doi.org/10.1038/nrclinonc.2017.43 (2017).

4. Drake, C. G., Lipson, E. J. \& Brahmer, J. R. Breathing new life into immunotherapy: review of melanoma, lung and kidney cancer. Nat. Rev. Clin. Oncol. 11, 24-37. https://doi.org/10.1038/nrclinonc.2013.208 (2014).

5. Kandolf Sekulovic, L. et al. More than 5000 patients with metastatic melanoma in Europe per year do not have access to recommended first-line innovative treatments. Eur. J. Cancer 75, 313-322. https://doi.org/10.1016/j.ejca.2017.01.012 (2017).

6. Larkin, J. et al. Combined nivolumab and ipilimumab or monotherapy in untreated melanoma. N. Engl. J. Med. 373, 23-34. https ://doi.org/10.1056/NEJMoa1504030 (2015).

7. Gershenwald, J. E. et al. Melanoma staging: evidence-based changes in the American Joint Committee on Cancer eighth edition cancer staging manual. CA Cancer J. Clin. 67, 472-492. https://doi.org/10.3322/caac.21409 (2017).

8. Weide, B. et al. Serum markers lactate dehydrogenase and S100B predict independently disease outcome in melanoma patients with distant metastasis. Br. J. Cancer 107, 422-428. https://doi.org/10.1038/bjc.2012.306 (2012).

9. Gershenwald, J. E. \& Scolyer, R. A. Melanoma staging: American Joint Committee on Cancer (AJCC) 8th edition and beyond. Ann. Surg. Oncol. 25, 2105-2110. https://doi.org/10.1245/s10434-018-6513-7 (2018).

10. Pos, O., Biro, O., Szemes, T. \& Nagy, B. Circulating cell-free nucleic acids: characteristics and applications. Eur. J. Hum. Genet. https://doi.org/10.1038/s41431-018-0132-4 (2018).

11. Gangadhar, T. C. et al. Feasibility of monitoring advanced melanoma patients using cell-free DNA from plasma. Pigment Cell Melanoma Res. https://doi.org/10.1111/pcmr.12623 (2017). 
12. Barbany, G. et al. Cell-free tumour DNA testing for early detection of cancer-a potential future tool. J. Intern. Med. 286, 118-136. https://doi.org/10.1111/joim.12897 (2019).

13. Haber, D. A. \& Velculescu, V. E. Blood-based analyses of cancer: circulating tumor cells and circulating tumor DNA. Cancer Discov. 4, 650-661. https://doi.org/10.1158/2159-8290.CD-13-1014 (2014).

14. Bidard, F. C., Weigelt, B. \& Reis-Filho, J. S. Going with the flow: from circulating tumor cells to DNA. Sci. Transl. Med. 5, 207-214. https://doi.org/10.1126/scitranslmed.3006305 (2013).

15. Ma, M. et al. "Liquid biopsy"-ctDNA detection with great potential and challenges. Ann. Transl. Med. 3, 235. https://doi. org/10.3978/j.issn.2305-5839.2015.09.29 (2015).

16. Wan, J. C. M. et al. Liquid biopsies come of age: towards implementation of circulating tumour DNA. Nat. Rev. Cancer 17, $223-238$. https://doi.org/10.1038/nrc.2017.7 (2017).

17. Rowe, S. P. et al. From validity to clinical utility: the influence of circulating tumor DNA on melanoma patient management in a real-world setting. Mol. Oncol. 12, 1661-1672. https://doi.org/10.1002/1878-0261.12373 (2018).

18. McEvoy, A. C. et al. Correlation between circulating tumour DNA and metabolic tumour burden in metastatic melanoma patients. BMC Cancer 18, 726. https://doi.org/10.1186/s12885-018-4637-6 (2018).

19. Gray, E. S. et al. Circulating tumor DNA to monitor treatment response and detect acquired resistance in patients with metastatic melanoma. Oncotarget 6, 42008-42018. https://doi.org/10.18632/oncotarget.5788 (2015).

20. Reid, A. L., Freeman, J. B., Millward, M., Ziman, M. \& Gray, E. S. Detection of BRAF-V600E and V600K in melanoma circulating tumour cells by droplet digital PCR. Clin. Biochem. 48, 999-1002. https://doi.org/10.1016/j.clinbiochem.2014.12.007 (2015).

21. Wong, S. Q. Circulating tumor DNA analysis and functional imaging provide complementary approaches for comprehensive disease monitoring in metastatic melanoma. Precis. Oncol. 1, 1-14 (2017).

22. Lee, J. H. et al. Circulating tumour DNA predicts response to anti-PD1 antibodies in metastatic melanoma. Ann. Oncol. 28, 1130-1136. https://doi.org/10.1093/annonc/mdx026 (2017).

23. Lee, R. J. et al. Circulating tumor DNA predicts survival in patients with resected high-risk stage II/III melanoma. Ann. Oncol. 29, 490-496. https://doi.org/10.1093/annonc/mdx717 (2018).

24. Cancer Genome Atlas, N. Genomic classification of cutaneous melanoma. Cell 161, 1681-1696. https://doi.org/10.1016/j. cell.2015.05.044 (2015).

25. Buder-Bakhaya, K., Machiraju, D. \& Hassel, J. C. Liquid biopsy: value for melanoma therapy. Oncol. Res. Treat. 40, 430-434. https ://doi.org/10.1159/000478893 (2017).

26. Huang, F. W. et al. Highly recurrent TERT promoter mutations in human melanoma. Science 339, 957-959. https://doi.org/10.1126/ science.1229259 (2013).

27. Ekedahl, H. et al. High TERT promoter mutation frequency in non-acral cutaneous metastatic melanoma. Pigment Cell Melanoma Res. 29, 598-600. https://doi.org/10.1111/pcmr.12500 (2016).

28. Vicente, A. et al. Mutational profile of driver genes in Brazilian melanomas. J. Glob. Oncol. 5, 1-14. https://doi.org/10.1200/ JGO.19.00169 (2019).

29. Nagore, E. et al. TERT promoter mutations associate with fast-growing melanoma. Pigment Cell Melanoma Res. 29, 236-238. https ://doi.org/10.1111/pcmr.12441 (2016).

30. Nagore, E. et al. TERT promoter mutations in melanoma survival. Int. J. Cancer 139, 75-84. https://doi.org/10.1002/ijc.30042 (2016).

31. Armbruster, D. A. \& Pry, T. Limit of blank, limit of detection and limit of quantitation. Clin Biochem. Rev. 29(Suppl 1), S49-52 (2008).

32. Huynh, K. \& Hoon, D. S. Liquid biopsies for assessing metastatic melanoma progression. Crit. Rev. Oncog. 21, 141-154. https:// doi.org/10.1615/CritRevOncog.2016016075 (2016).

33. Busser, B. et al. Plasma circulating tumor DNA levels for the monitoring of melanoma patients: landscape of available technologies and clinical applications. Biomed. Res. Int. 2017, 5986129. https://doi.org/10.1155/2017/5986129 (2017).

34. Gorgannezhad, L., Umer, M., Islam, M. N., Nguyen, N. T. \& Shiddiky, M. J. A. Circulating tumor DNA and liquid biopsy: opportunities, challenges, and recent advances in detection technologies. Lab Chip 18, 1174-1196. https://doi.org/10.1039/C8LC00100F (2018).

35. Postel, M., Roosen, A., Laurent-Puig, P., Taly, V. \& Wang-Renault, S. F. Droplet-based digital PCR and next generation sequencing for monitoring circulating tumor DNA: a cancer diagnostic perspective. Expert Rev. Mol. Diagn. 18, 7-17. https://doi. org/10.1080/14737159.2018.1400384 (2018).

36. Chen, M. \& Zhao, H. Next-generation sequencing in liquid biopsy: cancer screening and early detection. Hum Genom. 13, 34. https://doi.org/10.1186/s40246-019-0220-8 (2019).

37. Elazezy, M. \& Joosse, S. A. Techniques of using circulating tumor DNA as a liquid biopsy component in cancer management. Comput. Struct. Biotechnol. J. 16, 370-378. https://doi.org/10.1016/j.csbj.2018.10.002 (2018).

38. de Unamuno Bustos, B. et al. Towards personalized medicine in melanoma: implementation of a clinical next-generation sequencing panel. Sci Rep 7, 495. https://doi.org/10.1038/s41598-017-00606-w (2017).

39. Diefenbach, R. J. et al. Design and testing of a custom melanoma next generation sequencing panel for analysis of circulating tumor DNA. Cancers (Basel) https://doi.org/10.3390/cancers12082228 (2020).

40. Calapre, L. et al. Locus-specific concordance of genomic alterations between tissue and plasma circulating tumor DNA in metastatic melanoma. Mol. Oncol. 13, 171-184. https://doi.org/10.1002/1878-0261.12391 (2019).

41. McEvoy, A. C. et al. Sensitive droplet digital PCR method for detection of TERT promoter mutations in cell free DNA from patients with metastatic melanoma. Oncotarget 8, 78890-78900. https://doi.org/10.18632/oncotarget.20354 (2017).

42. Corless, B. C. et al. Development of novel mutation-specific droplet digital PCR assays detecting TERT promoter mutations in tumor and plasma samples. J. Mol. Diagn. 21, 274-285. https://doi.org/10.1016/j.jmoldx.2018.09.003 (2019).

43. Braune, J. et al. Circulating tumor DNA allows early treatment monitoring in BRAF- and NRAS-mutant malignant melanoma. JCO Precis. Oncol. 4, 20-31. https://doi.org/10.1200/PO.19.00174 (2020).

44. Calapre, L. et al. Locus-specific concordance of genomic alterations between tissue and plasma circulating tumor DNA in metastatic melanoma. Mol. Oncol. https://doi.org/10.1002/1878-0261.12391 (2018).

45. Sanmamed, M. F. et al. Quantitative cell-free circulating BRAFV600E mutation analysis by use of droplet digital PCR in the follow-up of patients with melanoma being treated with BRAF inhibitors. Clin. Chem. 61, 297-304. https://doi.org/10.1373/clinc hem.2014.230235 (2015).

46. Chang, G. A. et al. Sensitivity of plasma BRAFmutant and NRASmutant cell-free DNA assays to detect metastatic melanoma in patients with low RECIST scores and non-RECIST disease progression. Mol. Oncol. 10, 157-165. https://doi.org/10.1016/j.molon c.2015.09.005 (2016).

47. Schreuer, M. et al. Quantitative assessment of BRAF V600 mutant circulating cell-free tumor DNA as a tool for therapeutic monitoring in metastatic melanoma patients treated with BRAF/MEK inhibitors. J. Transl. Med. 14, 95. https://doi.org/10.1186/s1296 7-016-0852-6 (2016).

48. Campanella, N. C. et al. Mutational profiling of driver tumor suppressor and oncogenic genes in Brazilian malignant pleural mesotheliomas. Pathobiology 87, 208-216. https://doi.org/10.1159/000507373 (2020). 


\section{Acknowledgements}

This research was funded by grants from the Public Ministry of Labor Campinas (Research, Prevention, and Education of Occupational Cancer) and FAPESP (2018/14468-8). A sincere thanks to Jeremy Squire, for his diligent proofreading of this paper and scientific contribution. We would like to thank to Barretos Cancer Hospital Biobank and the Department of Pathology for the technical support.

\section{Author contributions}

G.T.M recruited patients, performed experiments, wrote the main manuscript, performed statistical analysis, acquisition of clinical pathological data. A.C.L carried out the experiments, revised all results, and verified the analytical methods. M.B.R provided expert advice, planning, execution, and analysis of the extra experiments. R.M.R supervised the findings of this work, interpretation of data, and helped supervise the project. V.L.V conceived the original idea, supervised the project, and contributed to patient selection and follow-up. All authors provided critical feedback, helped shape the research, analysis, revised the manuscript, and approved the submitted version.

\section{Competing interests}

The authors declare no competing interests.

\section{Additional information}

Supplementary information is available for this paper at https://doi.org/10.1038/s41598-020-75792-1.

Correspondence and requests for materials should be addressed to V.d.V.

Reprints and permissions information is available at www.nature.com/reprints.

Publisher's note Springer Nature remains neutral with regard to jurisdictional claims in published maps and institutional affiliations.

(c) (i) Open Access This article is licensed under a Creative Commons Attribution 4.0 International cc) License, which permits use, sharing, adaptation, distribution and reproduction in any medium or format, as long as you give appropriate credit to the original author(s) and the source, provide a link to the Creative Commons licence, and indicate if changes were made. The images or other third party material in this article are included in the article's Creative Commons licence, unless indicated otherwise in a credit line to the material. If material is not included in the article's Creative Commons licence and your intended use is not permitted by statutory regulation or exceeds the permitted use, you will need to obtain permission directly from the copyright holder. To view a copy of this licence, visit http://creativecommons.org/licenses/by/4.0/.

(C) The Author(s) 2020 